\title{
Theoretical studies of equilibrium of disc coulter counter with adaptive suspension
}

\author{
Kokoshin Sergei Nikolaevich \\ Mechanics and Technology Institute \\ FSBEI HE Northern Trans-Ural SAU \\ Tyumen, Russia \\ kokoshinsn@gausz.ru
}

\author{
Kizurov Anatoly Sergeevich \\ Mechanics and Technology Institute \\ FSBEI HE Northern Trans-Ural SAU \\ Tyumen, Russia \\ Impossible_@mail.ru
}

\begin{abstract}
One of the main factors influencing the yield is the depth of seed sowing. Modern technology involves the use of disc seeders. But due to changes in the physical and mechanical properties of the soil, the force acting on the coulter when moving in the field causes the disk to move in the vertical plane, affecting the depth of the sowing. Equilibrium of the opener during operation can be ensured by using a flexible tubular element that is able to create a force compensating for the displacement of the coulter from the force of soil resistance. This mechanism allows the creation of an adaptive disc coulter, which provides the necessary depth in the seeding process. For a mathematical analysis of the equilibrium during working the coulter operation, we propose a model that takes into account the direction of the force action line and its variable value when the seeding depth is changed. The article presents graphical dependencies of the soil resistance force and the angle of inclination of the force action line to the horizon from the depth of the coulter stroke, obtained by the mathematical method. A comparative analysis of the existing and proposed models for calculating the coulter balance is carried out.
\end{abstract}

Keywords—seed drill, disc, depth, soil, equilibrium, uniformity.

\section{INTRODUCTION}

Modern energy-saving technologies include the use of disk seeders [1], which also allow working at higher speeds. But, due to the fact that the soil resistance force acting on the coulter is not constant, then the coulter oscillations in the vertical plane occur, which affect the unevenness of the depth of seed sowing [2]. In the designs of modern seeders, a mechanism is provided that tracks the change in the depth of sowing $[3,4]$, but in combination with the depth adjustment system of the coulter, it presents a rather complex construction and is not used in practice. Therefore, at the moment, for adjusting the depth in the coulter designs, springs are provided, the rigidity of which is selected according to the coulter's calculations for stability, depending on soil properties [5]. But in the process of seeding, the spring is not able to equalize the installed depth of the disk in the event of a change in the physical and mechanical properties of the soil.

In modern technologies, which are not applied in the technology of strip-till and no-till. Sowing machines used in such cases, provided with anchor coulters, which destroy the soil with compression deformation, which has a high energy intensity. If the technology does not use a disc coulter, then there are two sharpened rotating knives made of high-strength steel, then it is possible to achieve reduction of traction resistance and cutting of plant residues. The advantages of the disc coulter can also be attributed to the fact that, theoretically, the direction of the seeding can be considered identical with the depth of the opener that is used in sowing. The strength of the stubble field resistance is higher than the field prepared for sowing. Therefore, when using discs on a stubble background, a mechanism for stabilizing the depth of seed sowing is necessary.

Therefore, we propose using a flexible tubular element in the construction of the disc coulter suspension, which changes its rigidity to bending, depending on the fluid pressure, fed into its internal cavity [6]. These elements have already been used during operation in the regime of power load [7] and soil treatment [8], which confirms their efficiency and working capacity.

\section{THEORETICAL BACKGROUND}

To maintain the established depth of go of the disc coulter with the proposed suspension on different types of soils, it is necessary to establish the relationship between the soil resistance force and the force created by the flexible tubular element under pressure (Fig. 1).

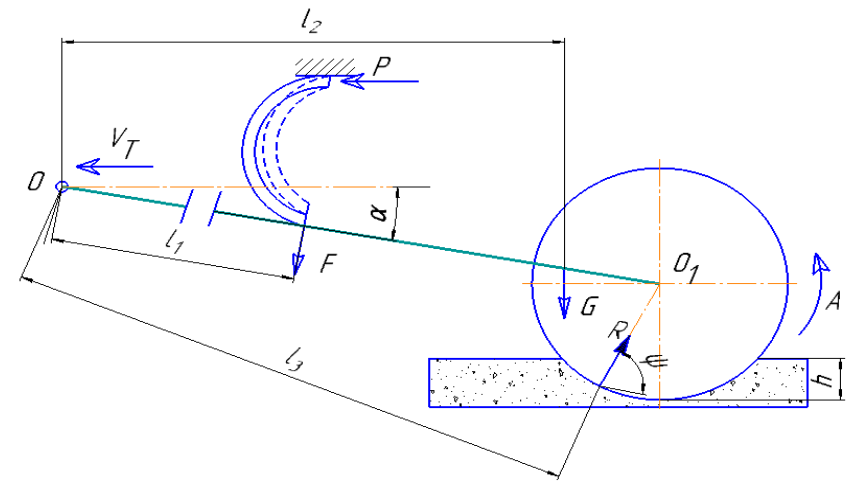

Fig. 1. Calculation scheme equilibrium of the coulter

If we consider the coulter and soil system in the first approximation, we can distinguish the following forces: the soil resistance $R$, the gravity power of the disc coulter with the leash $G$, force of the elastic element $F$. Before starting work, the disc is set to a depth of $h$ due to the forces of gravity and 
the elastic element. When the unit moves with a speed $V_{T}$, a resistance force acts from the soil side and tries to move the coulter in the direction "A", reducing the depth of sowing. To return the coulter to the set depth $h$, the liquid under pressure is injected into the cavity of the tubular element, deforming its cross section, thereby creating a force $F$ that moves the disk in the direction opposite to "A".

In order for the depth of the coulter to be constant, it is necessary that the following condition be satisfied:

$$
\Sigma M_{0}=0
$$

We substitute the moments of forces acting on the coulter into the left side of equation 1 :

$$
R \cdot l_{3}-G \cdot l_{2}-P \cdot l_{1}=0
$$

The action line of the resultant force soil resistance $R$ passes through the center of the coulter disc $O_{l}$ and is located at an angle $\psi$ with respect to the horizon [9]. In the calculations, it is not taken into account that when the depth of the coulter stroke changes, the application point and the angle of inclination of the force vector change. Therefore, we propose a model of interaction between the disc coulter and the soil, taking into account these parameters (Fig. 2, a).

The part of the soil interacting with the disk is a figure of OBD and exerts an effect in the form of a distributed load with a resultant $R$ applied at the center of gravity - the point $\mathrm{C}$ of this figure. When the depth of the disk changes, the coordinates of point $\mathrm{C}$ are shifted by changing the angle $\psi$. We express the coordinates of the point $\mathrm{D}$ through the angle of the sector $\varphi$, taking the point $\mathrm{O}$ as the origin:

$$
X_{D}=r \cdot \sin \varphi ; Y_{D}=r-r \cdot \cos \varphi,
$$

where $r$-the radius of the coulter.

We express the angle $\varphi$ in terms of the coordinate $\mathrm{Y}_{\mathrm{D}}$, which characterizes the depth of sowing, and substitute into the equation $\mathrm{X}_{\mathrm{D}}$ :

$$
\begin{gathered}
\varphi=\arccos \left(1-Y_{D} / r\right) . \\
X_{D}=r \cdot \sin \left(\arccos \left(1-Y_{D} / r\right)\right) .
\end{gathered}
$$

To determine the coordinate of the point $\mathrm{C}$, it is necessary to use the static moment of the figure [10]. To do this, let's represent the area of the ODB figure as the difference between the areas of the triangle ODB and the OD segment (Fig. 2, b) and express the desired coordinates through the characteristics of these figures:

$$
X_{C}=\Sigma S y / \Sigma A ; Y_{C}=\Sigma S x / \Sigma A,
$$

where $\Sigma S y, \Sigma S x$ - the sumes of the static moments of a triangle and a segment, $\mathrm{mm}^{3}$.

a)

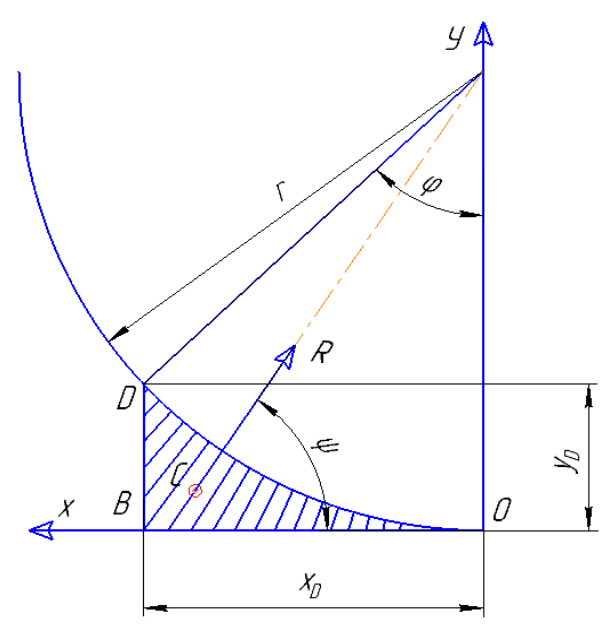

b)

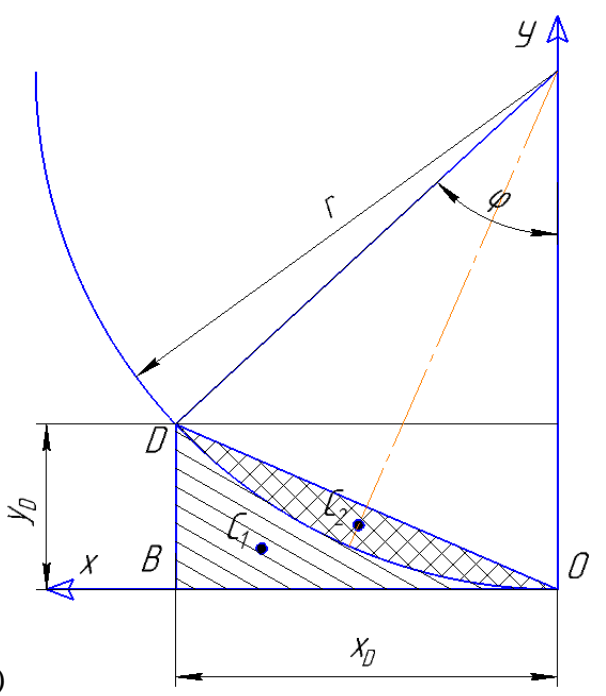

a - calculation scheme; $b$ - definition of the center of the figure.

Fig. 2. Model of disk interaction with soil

Let us define the center of gravity of the figure ODB, substituting the coordinates and the area of the presented figures:

$$
X_{C}=\left(X_{I} \cdot A_{1}-X_{2} \cdot A_{2}\right) /\left(A_{I}-A_{2}\right) ; Y_{C}=\left(Y_{1} \cdot A_{I}-Y_{2} \cdot A_{2}\right) /\left(A_{1}-A_{2}\right),
$$

where $X_{1}, Y_{1}, A_{1}$ - coordinates and area of the triangle;

$X_{2}, Y_{2}, A_{2}$ - coordinates and area of the segment.

To solve the system of equations, an algorithm was compiled in the mathematical package MathCAD. To solve the system, let us set the following parameters: soil resistivity during sowing $k=5 \times 10^{-2} \mathrm{MPa}$, disc coulter width $b=150$ $\mathrm{mm}$; the diameter of the disc coulter is $120 \mathrm{~mm}$. Fig. 3 shows the dependence of the change in the angle of inclination of the 
resultant of soil resistance force to the horizon from the depth of the coulter stroke.

It can be seen from Fig. 3 that when the coulter is buried, the angle $\psi$ decreases, changing the values of the projection of the soil drag force on the axes OX and OY:

$$
R_{X}=R \cdot \cos \psi ; R_{Y}=R \cdot \sin \psi,
$$

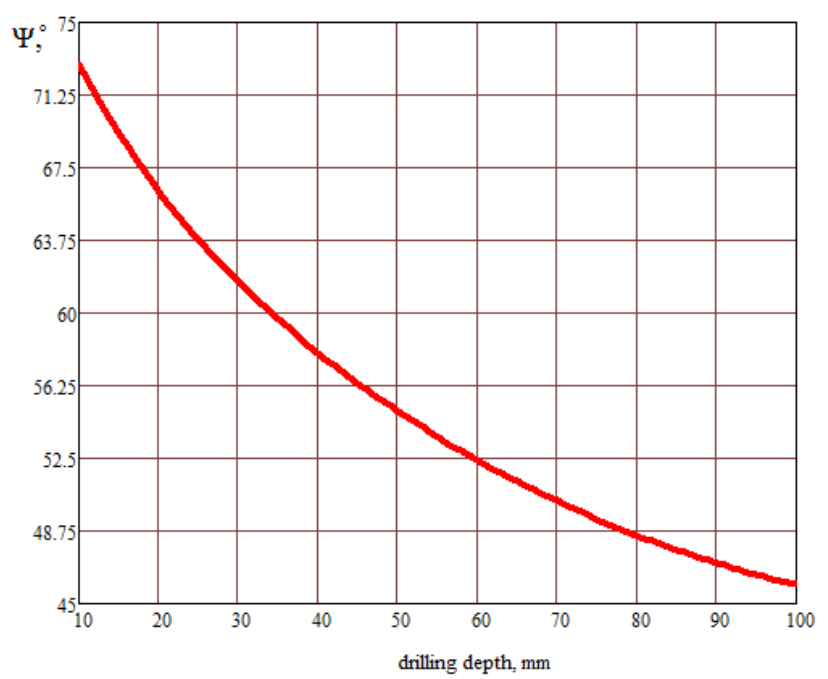

Fig. 3. Change in the angle of inclination of the soil resistance force. Example of a figure caption. (figure caption)

Analyzing equation 8, it turns out that a decrease in the angle $\psi$ leads to a more intensive growth of the horizontal projection of the soil resistance force than the vertical one.

Graphically this is shown in Fig. 4.

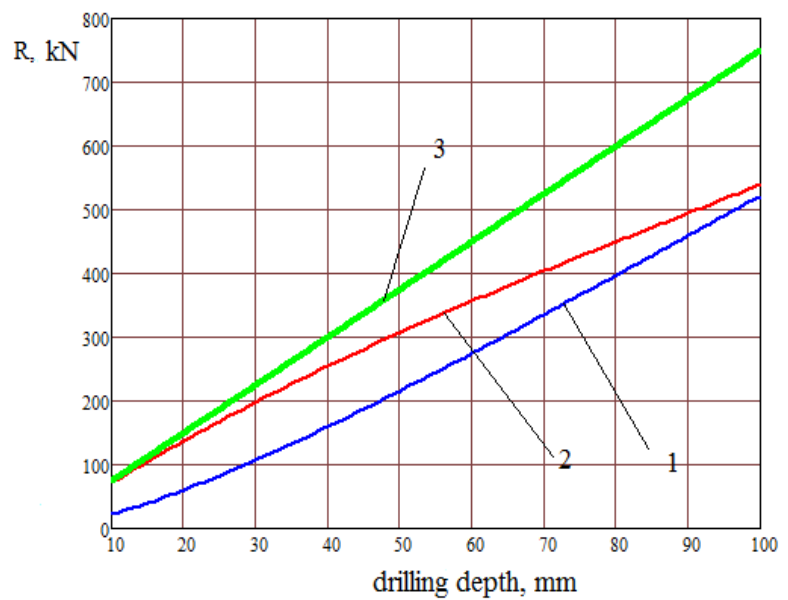

1 - force projection $R x ; 2$ - force projection $R y ; 3$ - force $R$

Fig. 4. Dependence of the force projections of soil resistance on the depth of the coulter

When calculating the equilibrium of the coulter, it must also be taken into account that the change in the angle $\psi$ affects the arm $l_{3}$ (Fig. 1), and therefore on the instant of the force $R$ relative to the hinge $O$. If the slope angle of the arm is $\alpha$, then the force moment can be determined from the equation:

$$
M(R)_{0}=R \cdot l_{3}=R \cdot O O_{1} \cdot \cos (\alpha+\psi),
$$

where $O O_{1}$-the length arm of the coulter, $\mathrm{m}$.

We perform a comparative analysis of the force moment values, provided that the force $R$ is always perpendicular to the leash (according to the existing procedure $-M_{l}$ ) and in the case when the force $R$ changes the point of application and the angle of inclination at the depth (the proposed procedure is $M_{2}$ ). The system of equations was solved in the mathematical package MathCAD, and the results are presented in the form of graphs in Fig. 5

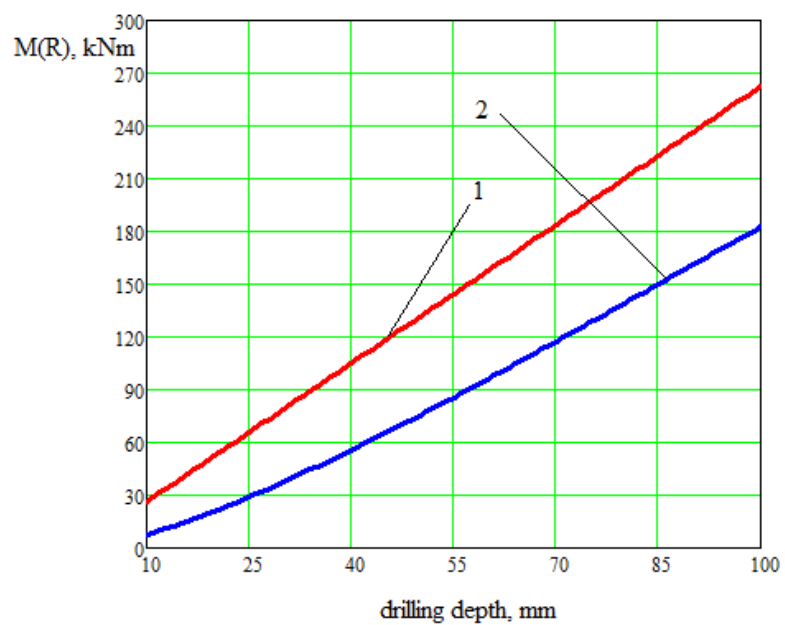

$1-M_{1}, 2-M_{2}$

Fig. 5. Value of the bending moment from the force of the resistance of the soil

\section{CONCLUSION}

From the obtained dependences it is seen that the moment of force relative to the arm of the coulter leash, calculated according to the proposed procedure, has smaller values. This is due to the fact that when the coulter is buried, the horizontal component of the soil resistance force increases more intensively than the vertical one, but having a small shoulder and the hinge has a negligible effect on the depth of the sowing. The initial adjustment of the spring of the coulters leads to an increase in seeding depth and increased fuel consumption. Therefore, the use of a flexible tubular element will allow the creation of an adaptive coulter suspension that will compensate deviations in the established depth from the soil resistance forces. Having determined the values of the moments of the force of soil resistance (Fig. 5), it is possible to calculate the value of the necessary force $F$ (Fig. 1), which will provide the established depth, according to equations 1,2. 


\section{References}

[1] E.V. Priporov, "Analysis of grain seeders with disc coulters for a minimal seeding technology," Electronic scientific journal, No. 8 (11). pp. 43-47, 2016

[2] Liu, Tao, Li, Rui, Jin, Xiuliang and others, "Evaluation of Seed Emergence Uniformity of Mechanically Sown Wheat with UAV RGB Imagery," Remote sensing, Vol. 9, Issue 12, Number of the article 1241, 2017.

[3] Nielse, S. Kirkegaard, Munkholm, L. Juhl, Lamande, Mathieu and others, "Seed drill depth control system for precision seeding," Computers and electronics in agriculture, Vol. 144, pp.174-180, 2018.

[4] E.V. Demchuk, A.S. Soyunov, V.V. Myalo, M.S. Chekusov, "Determination of the uniformity of seed distribution with a double-vane coulter with the help of software," Achievements of science and technology of agroindustrial complex, Vol. 29, No. 8, pp.69-71, 2015.
[5] V.V. Blednykh Design, "Calculation and design of tillage tools," Chelyabinsk: ChSAA, p.203, 2010.

[6] S. Kokoshin, B. Kirghintsev, "Disc coulter suspension mechanism," pat. RF on the utility model № 164384, Bul. № 24, 4, August 2016 .

[7] S. Kokoshin, N. Ustinov, B. Kirgincev "The use of flexible tubular elements of the overhaul and tunnels reconstruction," Procedia engineering, Vol. 165, pp. 817-828, 2016.

[8] N. Ustinov, A. Maratkanov, A. Martynenko, "Experimental study of the parameters of the active tool of a cultivator with a frame in form a flexible tubular element," International Science Conference, $\mathrm{SPb}$ WOSCE-2016 "SMART City", MATEC Web of Conferences, vol. 106, 08063, 2017.

[9] B.M. Khalansky, I.V. Gorbachev, Agricultural machines, St. Petersburg: Quadro, 2014, p.624.

[10] E.G. Kirsanova, Resistance of materials [Electronic resource]: Electron. text data. - Saratov: Ai Pi Er Media, 2012, p.110. 